\title{
Age range estimation based on facial wrinkle analysis using Hessian based filter
}

\begin{abstract}
Aging is a normal process that has an effect on different parts of the human body under the influence of various biological and environmental aspects. The most prominent changes that occur on the face are the form of the skin wrinkles, which are the main objective of this research. Specific wrinkle detection is an important task in face textural analysis. Previously, some researchers have been proposed the age range estimation based on wrinkle analysis in literature, but poor localization limits the performance of the whole age estimation process. This is because, when less number of wrinkles are detected or extracted, it will consequently affect the process to estimate the correct age. Therefore, we address this issue to enhance age range estimation method using a new approach to extract correct facial wrinkles for further analysis. We propose a method to extract facial wrinkle in face image using Hessian based filter $(\mathrm{HBF})$ for age estimation. In other word, this research focus on age range estimation method based on facial wrinkle analysis extracted from facial image obtained from FG-NET database using Hessian based filter. The proposed filter is theoretically straight forward, however, it significantly increases the wrinkle analysis result compared to previous methods. The result shows that HBF successfully obtained higher accuracy with over $90 \%$ estimation rate.
\end{abstract}

Keyword: Age range estimation; Wrinkle analysis; Wrinkle density; Hessian based filter 